PROCEEDINGS OF THE

AMERICAN MATHEMATICAL SOCIETY

Volume 134, Number 6, Pages 1605-1613

S 0002-9939(05)08157-8

Article electronically published on December 5, 2005

\title{
ON STABLE EQUIVALENCES INDUCED BY EXACT FUNCTORS
}

\author{
YUMING LIU
}

(Communicated by Martin Lorenz)

\begin{abstract}
Let $A$ and $B$ be two Artin algebras with no semisimple summands. Suppose that there is a stable equivalence $\alpha$ between $A$ and $B$ such that $\alpha$ is induced by exact functors. We present a nice correspondence between indecomposable modules over $A$ and $B$. As a consequence, we have the following: (1) If $A$ is a self-injective algebra, then so is $B$; (2) If $A$ and $B$ are finite dimensional algebras over an algebraically closed field $k$, and if $A$ is of finite representation type such that the Auslander-Reiten quiver of $A$ has no oriented cycles, then $A$ and $B$ are Morita equivalent.
\end{abstract}

\section{INTRODUCTION}

Given two Artin algebras $A$ and $B$, we say that $A$ and $B$ are stably equivalent if their stable categories $A-\underline{\bmod }$ and $B-\underline{\bmod }$ are equivalent. In general, a stable equivalence need not be induced by exact functors between module categories. But in practice one often uses those induced by exact functors. It was first noted that stable equivalences induced by exact functors arise naturally in the block theory of finite groups, or more generally, for self-injective algebras ([3, 13]), and they play a substantial role in the representation theory of self-injective algebras ([3, 15, 11, 14, 15]). Recently, it was shown that such equivalences also occur frequently between general finite dimensional algebras $([7,8])$, and they possess many interesting properties ([4, 6, 7, 8, 16]).

An important aspect of studying such equivalences is to compare simple modules. By Rickard [12, 13, if there is a stable equivalence $\alpha$ between two self-injective algebras $A$ and $B$ such that $\alpha$ is induced from a derived equivalence (such a stable equivalence is induced by exact functors), then $A$ and $B$ have the same number of simple modules. However, it is not known in general whether a stable equivalence induced by exact functors preserves the number of simple modules. In [5], Linckelmann proved that a stable equivalence $\alpha$ of Morita type (note that every stable equivalence of Morita type is a stable equivalence induced by exact functors) between two self-injective algebras is lifted to a Morita equivalence if and only if $\alpha$ maps any simple $A$-module to a simple $B$-module. In [6], Linckelmann's theorem was generalized to arbitrary finite dimensional algebras. It is natural to ask

Received by the editors September 28, 2004 and, in revised form, January 11, 2005.

2000 Mathematics Subject Classification. Primary 16G10; Secondary 16G70.

Key words and phrases. Stable equivalence induced by exact functors, simple module, indecomposable module.

(C)2005 American Mathematical Society Reverts to public domain 28 years from publication 
whether there is some nice correspondence between simple modules under a stable equivalence induced by exact functors.

One of the main aims of this paper is to give an answer to the last question. In fact, we show even more: some nice correspondence between indecomposable modules under a stable equivalence induced by exact functors. Namely, we have the following

Theorem 1.1. Let $A$ and $B$ be two Artin algebras with no semisimple summands. Let $F: A$-mod $\rightarrow B$-mod and $G: B$-mod $\rightarrow A$-mod be two exact functors. Suppose that $F$ and $G$ induce the stable equivalences $F^{\prime}: A-\underline{\bmod } \rightarrow B$ - $\underline{\bmod }$ and $G^{\prime}: B$ $\underline{\bmod } \rightarrow A$ - $\underline{\bmod }$ such that $F^{\prime}$ is the quasi-inverse of $G^{\prime}$. Denote by $H^{\prime}=D \operatorname{tr} F^{\prime} \operatorname{tr} D$ : $A$-mod $\rightarrow B$-mod the induced equivalence. Then there is a bijection $\phi: \operatorname{ind}(A) \backslash$ $P I \rightarrow \operatorname{ind}(B) \backslash P I$ given by

$$
\phi(X)=\left\{\begin{array}{l}
H^{\prime}(X) \quad \text { if } X \text { is projective, } \\
F^{\prime}(X) \quad \text { if } X \text { is non-projective, }
\end{array}\right.
$$

where ind $(A)$ and $P I$ denote the isomorphism classes of indecomposable A-modules and its subclasses of projective-injective modules, respectively. In particular, $\phi$ preserves projective modules, simple projective modules, injective modules, simple injective modules and nodes.

Note that Theorem 1.1 implies that a stable equivalence induced by exact functors preserves the number of simple modules if and only if it preserves the number of non-projective simple modules.

As a direct consequence of Theorem 1.1, we get that the class of self-injective algebras is closed under stable equivalences induced by exact functors.

As another consequence, we recall some results in [10, where Martinez-Villa considered algebras stably equivalent to factors of hereditary algebras and proved the following: if $\Lambda$ and $\Gamma$ are stably equivalent finite dimensional algebras over an algebraically closed field $k$ such that $\Lambda$ and $\Gamma$ have no nodes and have no semisimple summands, and that $\Lambda$ is of finite representation type and the Auslander-Reiten quiver of $\Lambda$ has no oriented cycles, then $\Lambda$ and $\Gamma$ are Morita equivalent. Combining methods in 10, with Theorem 1.1, we know that, for stable equivalences induced by exact functors, the above condition "no nodes" can be removed.

\section{Preliminaries}

Let $R$ be a commutative Artin ring. Recall from [2] that an $R$-algebra $A$ is called an Artin algebra if $A$ is finitely generated as a $R$-module. Important examples of Artin algebras are finite dimensional algebras over a field.

Given an Artin algebra $A$, we denote by $A$-mod the category of all finitely generated left $A$-modules. The socle of an $A$-module $X$, denoted by $\operatorname{soc}(X)$, is defined to be the submodule of $X$ generated by all semisimple submodules of $X$. The $\operatorname{rad}-$ ical of $X$ is the intersection of all the maximal submodules of $X$, and is written by $\operatorname{rad}(M)$. The composition of two morphisms $f: X \rightarrow Y$ and $g: Y \rightarrow Z$ is a morphism from $X$ to $Z$, which will be denoted by $f g$.

The stable category $A$-mod of $A$ is defined as follows: The objects of $A$-mod are the same as those of $A$-mod, and the morphisms between two objects $X$ and $Y$ are given by the quotient $R$-module $\underline{\operatorname{Hom}}_{A}(X, Y)=\operatorname{Hom}_{A}(X, Y) / \mathcal{P}(X, Y)$, where $\mathcal{P}(X, Y)$ is the $R$-submodule of $\operatorname{Hom}_{A}(X, Y)$ consisting of those homomorphisms 
from $X$ to $Y$ which factor through a projective $A$-module. Similarly, we have a stable category $A-\overline{m o d}$ of $A$ which is obtained by modulo injective modules.

Given two Artin algebras $A$ and $B$, we say that $A$ and $B$ are stably equivalent if their stable categories $A-\underline{\bmod }$ and $B-\underline{m o d}$ are equivalent. If two Artin algebras $A$ and $B$ are stably equivalent, we have the following properties which are taken from [2, Chapter X].

Lemma 2.1. Let $\alpha: A-\underline{\bmod } \rightarrow B-\underline{\bmod }$ be a stable equivalence. Then we have the following:

(1) $\alpha$ induces a one-to-one correspondence between the isomorphism classes of indecomposable non-projective modules in A-mod and those in B-mod (for convenience we shall also denote this correspondence by $\alpha$ ).

(2) The induced functor $\beta=D \operatorname{tr} \alpha \operatorname{tr} D: A-\overline{\bmod } \rightarrow B-\overline{\bmod }$ is an equivalence, where Dtr and $\operatorname{tr} D$ denote the Auslander-Reiten translation and its inverse, respectively.

In this paper, we are interested in stable equivalences which are induced by exact functors between module categories. For example, the stable equivalences of Morita type between finite dimensional algebras introduced by Broué [3] are stable equivalences induced by exact functors. Note that for self-injective algebras, stable equivalences induced by exact functors coincide with stable equivalences of Morita type by Rickard [14, theorem 3.2].

The basic tool in this paper is the theory of almost split sequences, so let us recall some fundamental notions on almost split sequences (see [2]).

Given an Artin algebra $A$, let $f: Y \rightarrow Z$ and $g: X \rightarrow Y$ be morphisms in $A$-mod. $f$ is right almost split if $f$ is not a split epimorphism, and any morphism $U \rightarrow Z$ which is not a split epimorphism factors through $f$. Dually, $g$ is left almost split if $g$ is not a split monomorphism, and any morphism $X \rightarrow V$ which is not a split monomorphism factors through $g$.

An exact sequence $0 \rightarrow X \stackrel{g}{\rightarrow} Y \stackrel{f}{\rightarrow} Z \rightarrow 0$ in $A$-mod is called an almost split sequence (or an Auslander-Reiten sequence) if $g$ is left almost split and $f$ is right almost split. Auslander and Reiten proved that, for any indecomposable nonprojective module $Z$ in $A$-mod, there is an almost split sequence (unique up to isomorphism) of the following form:

$$
0 \rightarrow \operatorname{Dtr}(Z) \rightarrow Y \rightarrow Z \rightarrow 0,
$$

where $\operatorname{Dtr}(Z)$ is the Auslander-Reiten translation of $Z$, and dually, for any indecomposable non-injective module $X$ in $A$-mod, there is an almost split sequence (unique up to isomorphism) of the following form:

$$
0 \rightarrow X \rightarrow Y \rightarrow \operatorname{tr} D(X) \rightarrow 0,
$$

where $\operatorname{tr} D(X)$ is the inverse of the Auslander-Reiten translation of $X$.

In what follows we freely use all properties of almost split sequences which can be found in [2].

\section{Proof of the main Result}

Throughout this section, we assume that $A$ and $B$ are two Artin algebras with no semisimple summands, and that $F: A$-mod $\rightarrow B$-mod and $G: B$-mod $\rightarrow A$-mod are two exact functors which induce the stable equivalences $F^{\prime}: A-\underline{\bmod } \rightarrow B-\underline{\bmod }$ and $G^{\prime}: B$ - $\underline{\bmod } \rightarrow A$ - $\underline{\bmod }$ such that $F^{\prime}$ is the quasi-inverse of $\overline{G^{\prime}}$. Clearly, in 
this case, $F$ and $G$ take projective modules to projective modules. By Lemma 2.1. $F^{\prime}$ induces a one-to-one correspondence between the isomorphism classes of indecomposable non-projective modules in $A$-mod and that in $B$-mod. In addition, we denote by $H^{\prime}=D \operatorname{tr} F^{\prime} \operatorname{tr} D: A-\overline{\bmod } \rightarrow B$ - $\overline{\bmod }$ the induced equivalence.

Before giving the proof of our result, we introduce the following notation. For an $A$-module $X$, we have a unique (up to isomorphism) decomposition $X=X^{\prime} \oplus P_{X}$, where $X^{\prime}$ has no non-zero projective summands and $P_{X}$ is projective. We call $P_{X}$ the projective part of $X$.

Lemma 3.1. $H^{\prime}$ induces a one-to-one correspondence between the isomorphism classes of simple projective modules in A-mod and that in B-mod.

Proof. Let $S$ be a simple projective $A$-module. Then $S$ is non-injective since $A$ has no semisimple summands. By [2, Proposition 2.6, p. 151], we have an almost split sequence $0 \longrightarrow S \longrightarrow E \longrightarrow \operatorname{tr} D S \longrightarrow 0$, where $E$ is projective. Applying $F$ we get an exact sequence $0 \longrightarrow F(S) \longrightarrow F(E) \longrightarrow F(\operatorname{tr} D S) \longrightarrow 0$ in $B$ mod, where $F(S)$ and $F(E)$ are projective. The above exact sequence induces canonically the following exact sequence: $0 \longrightarrow F(S) \longrightarrow F(E)_{0} \longrightarrow F^{\prime}(\operatorname{tr} D S) \longrightarrow$ 0 , where $F^{\prime}(\operatorname{tr} D S)$ is indecomposable non-projective, $F(E) \simeq F(E)_{0} \oplus P_{F(\operatorname{tr} D S)}$ and $P_{F(t r D S)}$ is the projective part of $F(\operatorname{tr} D S)$. By [2, Proposition 1.3, p. 337], we have an almost split sequence $0 \longrightarrow L \longrightarrow E^{\prime} \longrightarrow F^{\prime}(\operatorname{tr} D S) \longrightarrow 0$ in $B$-mod, where $E^{\prime}$ is projective. Since $E^{\prime}$ is projective and the morphism $F(E)_{0} \longrightarrow F^{\prime}(\operatorname{tr} D S)$ is not split, we have the following commutative diagram:

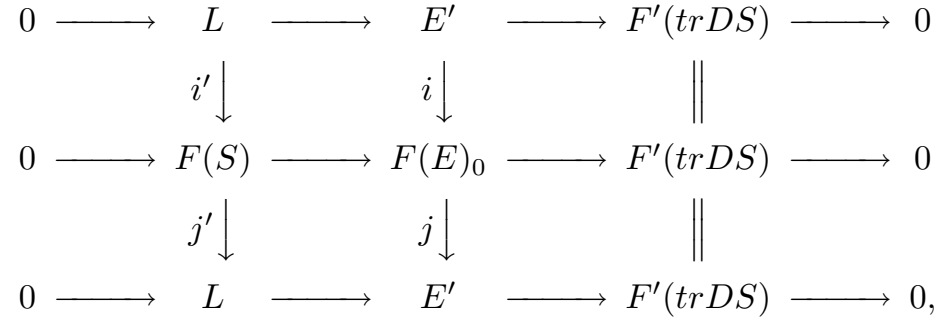

where the rows in the above diagram are all exact sequences. Since $E^{\prime} \longrightarrow F^{\prime}(\operatorname{tr} D S)$ is a right minimal almost split morphism, ij : $E^{\prime} \longrightarrow E^{\prime}$ is an isomorphism. It follows from the Five Lemma that $i^{\prime} j^{\prime}: L \longrightarrow L$ is an isomorphism. This implies that $L$ is a direct summand of $F(S)$ and therefore is a projective module. Since $E^{\prime}$ is projective, it follows from [2, Theorem 3.3, p. 154] that $L$ is a simple module. Thus $L$ is a simple projective $B$-module and $L \simeq D \operatorname{tr} F^{\prime} \operatorname{tr} D(S)=H^{\prime}(S)$.

We have proved that $H^{\prime}$ gives a map between the isomorphism classes of simple projective modules in $A$-mod and that in $B$-mod. Suppose that $S_{1}$ and $S_{2}$ are two non-isomorphic simple projective $A$-modules; then the simple $B$-modules $D \operatorname{tr} F^{\prime} \operatorname{tr} D\left(S_{1}\right) \not D \operatorname{tr} F^{\prime} \operatorname{tr} D\left(S_{2}\right)$ by the uniqueness of almost split sequences and Lemma 2.1. This shows that $H^{\prime}$ is an injective map. Similarly, we can prove that $D \operatorname{tr} G^{\prime} \operatorname{tr} D$ gives an injective map between the isomorphism classes of simple projective modules in $B$-mod and that in $A$-mod. This shows that $H^{\prime}$ is a bijection.

Lemma 3.2. $H^{\prime}$ induces a one-to-one correspondence between the isomorphism classes of indecomposable non-simple non-injective projective modules in A-mod and that in B-mod.

Proof. This was proved for stable equivalences in [1]. 
Recall from [9] that a simple non-projective non-injective module $S$ is called a node if the middle term $E$ of the almost split sequence $0 \longrightarrow S \longrightarrow E \longrightarrow \operatorname{tr} D S \longrightarrow$ 0 is projective.

Lemma 3.3. $F^{\prime}$ induces a one-to-one correspondence between the isomorphism classes of nodes in A-mod and that in B-mod.

Proof. Let $S$ be a node in $A$-mod. Then we have an almost split sequence $0 \longrightarrow$ $S \longrightarrow E \longrightarrow \operatorname{tr} D S \longrightarrow 0$, where $E$ is projective. Applying $F$ we get an exact sequence $0 \longrightarrow F(S) \longrightarrow F(E) \longrightarrow F(\operatorname{tr} D S) \longrightarrow 0$ in $B$-mod, where $F(E)$ is projective. The above exact sequence canonically induces the following exact sequence: $0 \longrightarrow F(S) \longrightarrow F(E)_{0} \longrightarrow F^{\prime}(\operatorname{tr} D S) \longrightarrow 0$, where $F^{\prime}(\operatorname{tr} D S)$ is indecomposable non-projective, $F(E) \simeq F(E)_{0} \oplus P_{F(\operatorname{trDS})}$ and $P_{F(\operatorname{tr} D S)}$ is the projective part of $F(\operatorname{tr} D S)$. By [2, Proposition 1.3, p. 337], we have an almost split sequence $0 \longrightarrow L \longrightarrow E^{\prime} \longrightarrow F^{\prime}(\operatorname{tr} D S) \longrightarrow 0$ in $B$-mod, where $E^{\prime}$ is projective. Since $E^{\prime}$ is projective and the morphism $F(E)_{0} \longrightarrow F^{\prime}(\operatorname{tr} D S)$ is not split, we have the following commutative diagram:

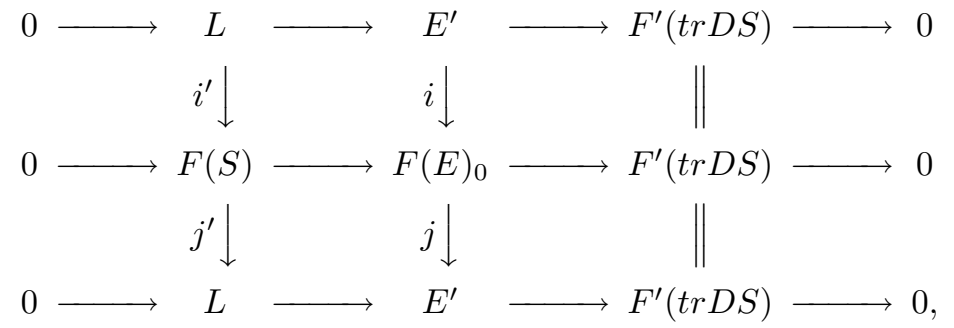

where the rows in the above diagram are all exact sequences. Since $E^{\prime} \longrightarrow F^{\prime}(\operatorname{tr} D S)$ is a right minimal almost split morphism, ij : $E^{\prime} \longrightarrow E^{\prime}$ is an isomorphism. It follows from the Five Lemma that $i^{\prime} j^{\prime}: L \longrightarrow L$ is an isomorphism. This implies that both $i$ and $i^{\prime}$ are split monomorphisms, and that $F(S) / i^{\prime}(L) \simeq F(E)_{0} / i\left(E^{\prime}\right)$ is a projective module by the Snake Lemma. Since $E^{\prime}$ is projective, it follows from [2, Theorem 3.3, p. 154] that $L$ is a simple non-injective module. $L$ is also a nonprojective module. Otherwise, $F(S) \simeq i^{\prime}(L) \oplus F(S) / i^{\prime}(L)$ is a projective module, which contradicts the fact that $S$ is non-projective. Thus $L$ is a node in $B$-module and $L \simeq F^{\prime}(S)$.

We have proved that $F^{\prime}$ gives a map between the isomorphism classes of nodes in $A$-mod and that in $B$-mod. Suppose that $S_{1}$ and $S_{2}$ are two non-isomorphic nodes in $A$-mod; then $F^{\prime}\left(S_{1}\right) ¥ F^{\prime}\left(S_{2}\right)$ by Lemma 2.1. This shows that $F^{\prime}$ is an injective map. Similarly, we can prove that $G^{\prime}$ gives an injective map between the isomorphism classes of nodes in $B$-mod and that in $A$-mod. This shows that $F^{\prime}$ is a bijection.

Remark. It was shown in 9 that for an Artin algebra $\Lambda$ with nodes, one can produce another Artin algebra $\Gamma$ without nodes such that $\Lambda$ and $\Gamma$ are stably equivalent. By Lemma 3.3. such a stable equivalence cannot be induced by exact functors.

Lemma 3.4. $F^{\prime}$ induces a one-to-one correspondence between the isomorphism classes of indecomposable non-projective non-injective modules in A-mod and that in $B$-mod. 
Proof. Let $X$ be an indecomposable non-projective non-injective module in $A$-mod. Then we have an almost split sequence $0 \longrightarrow X \longrightarrow E \longrightarrow \operatorname{tr} D X \longrightarrow 0$. There are two cases to be considered.

The first case: $X$ is a node. By Lemma 3.3, $F^{\prime}(X)$ is also a node.

The second case: $X$ is not a node. Then $E$ is not projective. By 2, Proposition 1.6, p. 339], we have an almost split sequence: $0 \longrightarrow F^{\prime}(X) \longrightarrow F^{\prime}(E) \oplus I \longrightarrow$ $F^{\prime}(\operatorname{tr} D X) \longrightarrow 0$ in $B$-mod, where $I$ is projective. This implies that $F^{\prime}(X)$ is an indecomposable non-projective non-injective module.

We have proved that $F^{\prime}$ gives a map between the isomorphism classes of indecomposable non-projective non-injective modules in $A$-mod and that in $B$-mod. Suppose that $X_{1}$ and $X_{2}$ are two non-isomorphic indecomposable non-projective non-injective modules in $A$-mod; then $F^{\prime}\left(X_{1}\right) \varsubsetneqq F^{\prime}\left(X_{2}\right)$ by Lemma 2.1. This shows that $F^{\prime}$ is an injective map. Similarly, we can prove that $G^{\prime}$ gives an injective map between the isomorphism classes of indecomposable non-projective non-injective modules in $B$-mod and that in $A$-mod. This shows that $F^{\prime}$ is a bijection.

Lemma 3.5. $F^{\prime}$ induces a one-to-one correspondence between the isomorphism classes of indecomposable non-projective injective modules in A-mod and that in B-mod.

Proof. By Lemma 2.1, $F^{\prime}$ induces a one-to-one correspondence between the isomorphism classes of indecomposable non-projective modules in $A$-mod and that in $B$-mod. By Lemma 3.4 $F^{\prime}$ induces a one-to-one correspondence between the isomorphism classes of indecomposable non-projective non-injective modules in $A$ mod and that in $B$-mod. It follows that $F^{\prime}$ induces a one-to-one correspondence between the isomorphism classes of indecomposable non-projective injective modules in $A$-mod and that in $B$-mod.

Lemma 3.6. $F^{\prime}$ induces a one-to-one correspondence between the isomorphism classes of simple injective modules in A-mod and that in B-mod.

Proof. Let $S$ be a simple injective $A$-module. Then $S$ is non-projective since $A$ has no semisimple summands. By Lemma 3.5. $F^{\prime}(S)$ is an injective module. By 2, Proposition 2.5, p. 151], we have an almost split sequence $0 \longrightarrow D \operatorname{tr} S \longrightarrow$ $E \longrightarrow S \longrightarrow 0$, where $E$ is injective. Consider the almost split sequence $0 \longrightarrow$ $\operatorname{DtrF}^{\prime}(S) \longrightarrow E^{\prime} \longrightarrow F^{\prime}(S) \longrightarrow 0$ in $B$-mod. We claim that $E^{\prime}$ is injective. Otherwise, $E^{\prime}$ has an indecomposable non-injective summand $E^{\prime \prime}$. If $E^{\prime \prime}$ is also non-projective, then we have an irreducible morphism $G^{\prime}\left(E^{\prime \prime}\right) \longrightarrow G^{\prime}\left(F^{\prime}(S)\right) \simeq S$. By Lemma 3.4, $G^{\prime}\left(E^{\prime \prime}\right)$ is non-injective. This contradicts the fact that $E$ is injective in the almost split sequence $0 \longrightarrow D \operatorname{tr} S \longrightarrow E \longrightarrow S \longrightarrow 0$ (see 2, Theorem 5.3, p. 167]). Thus $E^{\prime \prime}$ is projective non-injective and we have an irreducible morphism $H^{\prime-1}\left(\operatorname{Dtr}^{\prime}(S)\right) \simeq \operatorname{Dtr} S \longrightarrow H^{\prime-1}\left(E^{\prime \prime}\right)$. By Lemma3.1 and Lemma3.2, $H^{\prime-1}\left(E^{\prime \prime}\right)$ is projective non-injective. This contradicts the fact that $E$ is injective in the almost split sequence $0 \longrightarrow D \operatorname{tr} S \longrightarrow E \longrightarrow S \longrightarrow 0$ (see [2, Theorem 5.3, p. 167]). Hence $E^{\prime}$ is injective and $F^{\prime}(S)$ is simple injective by [2, Theorem 3.3, p. 154].

We have proved that $F^{\prime}$ gives a map between the isomorphism classes of simple injective modules in $A$-mod and that in $B$-mod. Suppose that $S_{1}$ and $S_{2}$ are two non-isomorphic simple injective modules in $A$-mod; then $F^{\prime}\left(S_{1}\right)$ and $F^{\prime}\left(S_{2}\right)$ are non-isomorphic in $B$-mod by Lemma 2.1. This shows that $F^{\prime}$ is an injective map. Similarly, we can prove that $G^{\prime}$ gives an injective map between the isomorphism 
classes of simple injective modules in $B$-mod and that in $A$-mod. This shows that $F^{\prime}$ is a bijection.

Proof of Theorem 1.1. This is a direct consequence of Lemma 3.1-Lemma 3.6.

As a consequence, we get that the class of self-injective algebras are closed under stable equivalences induced by exact functors.

Corollary 3.7. Let $A$ and $B$ be two Artin algebras (with no semisimple summands) such that $A$ is a self-injective algebra. Suppose that there is a stable equivalence $F^{\prime}$ between $A$ and $B$ such that $F^{\prime}$ is induced by exact functors. Then $B$ is also a self-injective algebra.

Proof. Note that for a self-injective algebra $A$, an $A$-module $X$ is projective if and only if it is injective. Suppose that $B$ is not self-injective. Then there is an indecomposable injective non-projective $B$-module. By Lemma 3.5, there is also an indecomposable injective non-projective $A$-module. This is a contradiction!

Recall from 10 that if $\alpha: \Lambda-\underline{\bmod } \rightarrow \Gamma-\underline{\bmod }$ is a stable equivalence between artin algebras $\Lambda$ and $\Gamma$ such that $\Lambda$ and $\Gamma$ have no semisimple summands and $\Lambda$ is a factor of hereditary algebra, then there is a one-to-one correspondence $\phi$ between the isomorphism classes of indecomposable projective-injective modules in $\Lambda$-mod and that in $\Gamma$-mod given by $\phi(P)=\underline{P}(\alpha(P / \operatorname{soc}(P)))$, where $\phi$ satisfies $\beta(\operatorname{rad}(P)) \simeq \operatorname{rad}(\phi(P))$ and $\alpha(P / \operatorname{soc}(P)) \simeq \phi(P) / \operatorname{soc}(\phi(P))$, and $\underline{P}(\alpha(P / \operatorname{soc}(P)))$ denotes the projective cover of $\alpha(P / \operatorname{soc}(P))$, and $\beta=D \operatorname{tr} \alpha \operatorname{tr} D$ denotes the induced equivalence.

Let $A$ and $B$ be as in Theorem 1.1. Suppose that $A$ is also a factor of hereditary algebra. Then the map $\phi$ in Theorem 1.1 can be extended as a bijection $\phi$ : $\operatorname{ind}(A) \rightarrow \operatorname{ind}(B)$ given by

$$
\phi(X)=\left\{\begin{array}{cc}
F^{\prime}(X) & \text { if } X \text { is non-projective, } \\
H^{\prime}(X) & \text { if } X \text { is projective non-injective, } \\
\underline{P}\left(F^{\prime}(X / \operatorname{soc}(X))\right) & \text { if } X \text { is projective-injective, }
\end{array}\right.
$$

where $\phi$ preserves: projective modules, simple projective modules, injective modules, simple injective modules, nodes, and projective-injective modules. Now using the same methods as Proposition 8-Theorem 13 in [10, we get the following consequence.

Corollary 3.8. Let $A$ and $B$ be finite dimensional algebras (with no semisimple summands) over an algebraically closed field $k$ such that $A$ is of finite representation type and the Auslander-Reiten quiver of A has no oriented cycles. If there is a stable equivalence $F^{\prime}$ between $A$ and $B$ such that $F^{\prime}$ is induced by exact functors, then $A$ and $B$ are Morita equivalent.

Remarks. (1) In particular, all the results obtained in this paper hold for stable equivalences of Morita type between finite dimensional algebras.

(2) It is easy to prove that a stable equivalence induced by exact functors preserves the finitistic dimension of Artin algebras (for further information on finitistic dimension, one may refer the recent paper [17] and the references therein). However, the following example shows that in general a stable equivalence between two Artin algebras is not induced by exact functors although they have the same finitistic dimension. 
Example. Let $k$ be a field. Let $\Lambda$ be the algebra given by the quiver $1 \stackrel{\alpha}{\longrightarrow}$ $2 \stackrel{\beta}{\longrightarrow} 3 \stackrel{\gamma}{\longrightarrow} 4$ with relation $\alpha \beta=0$, and let $\Gamma$ be the algebra given by the quiver $1^{\prime} \stackrel{\alpha^{\prime}}{\longrightarrow} 2^{\prime} \stackrel{\beta^{\prime}}{\longrightarrow} 3^{\prime} \stackrel{\gamma^{\prime}}{\longrightarrow} 4^{\prime}$ with relation $\beta^{\prime} \gamma^{\prime}=0$. Both $\Lambda$ and $\Gamma$ have finitistic dimension 2 .

We display the Auslander-Reiten quivers of $\Lambda$ and $\Gamma$ as follows:
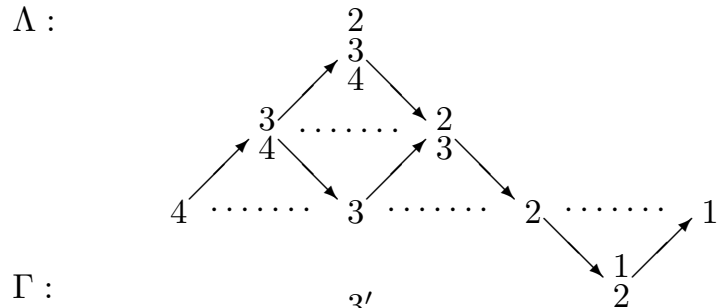

$\Gamma:$

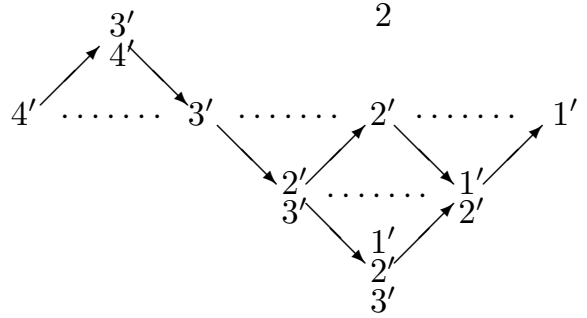

where the dotted lines indicate the Auslander-Reiten translation. $\Lambda$ and $\Gamma$ are derived equivalent since both $\Lambda$ and $\Gamma$ are tilted from the hereditary algebra $A_{4}$. Also $\Lambda$ and $\Gamma$ are stably equivalent since both $\Lambda$ and $\Gamma$ are stably equivalent to the hereditary algebra $A_{2} \times A_{3}$. However, a stable equivalence $\alpha$ between $\Lambda$ and $\Gamma$ cannot be induced by exact functors by Corollary 3.8 .

\section{ACKNOWLEDGEMENT}

I thank Professor Changchang Xi for his guidance and many helpful conversations. This research work was supported by CFKSTIP (No. 704004), and the Doctor Program Foundation (No. 20040027002), Ministry of Education of China.

\section{REFERENCES}

[1] M. Auslander and I. Reiten, Stable equivalence of Artin algebras. LNM 353, 1973, 8-71. MR0335575 (49:356)

[2] M. Auslander, I. Reiten and S.O. Smalø, Representation theory of Artin algebras. Cambridge University Press, 1995. MR1314422 (96c:16015)

[3] M. Broué, Equivalences of blocks of group algebras. In: Finite dimensional algebras and related topics. V. Dlab and L. L. Scott (eds.), Kluwer, 1994, 1-26. MR1308978 (97c:20004)

[4] H. Krause, Representation type and stable equivalences of Morita type for finite dimensional algebras. Math. Zeit. 229(1998), 601-606. MR1664779 (99k:16024)

[5] M. Linckelmann, Stable equivalences of Morita type for selfinjective algebras and $p$-groups. Math. Zeit. 223(1996), 87-100. MR1408864 (97j:20011)

[6] Y.M. LiU, On stable equivalences of Morita type for finite dimensional algebras. Proc. Amer. Math. Soc. 131(2003), 2657-2662. MR.1974320(2004a:16021)

[7] Y.M. LiU AND C.C. XI, Constructions of stable equivalences of Morita type for finite dimensional algebras I. Trans. Amer. Math. Soc. (to appear).

[8] Y.M. LiU AND C.C. XI, Constructions of stable equivalences of Morita type for finite dimensional algebras II. Math. Zeit. (to appear).

[9] R. Martinez-Villa, Algebras stably equivalent to $\ell$-hereditary. LNM 832, 1979, 396-431. MR0607166 (82b:16017) 
[10] R. Martinez-Villa, Algebras stably equivalent to factors of hereditary. LNM 903, 1981, 222-241. MR0654713 (83g:16057)

[11] Z. PogorzaLY, Invariance of Hochschild cohomology algebras under stable equivalences of Morita type. J. Math. Japan 53(2001), No. 4, 913-918. MR1852888(2002m:16007)

[12] J. RickARD, Morita theory for derived categories. J. London Math. Soc. 39(1989), 436-456. MR:1002456 (91b:18012)

[13] J. RiCKARD, Derived equivalences as derived functors. J. London Math. Soc. 43(1991), 37-48. MR:1099084 (92b:16043)

[14] J. RickARD, Some recent advances in modular representation theory. Canad. Math. Soc. Conf. Proc. 23(1998), 157-178. MR.1648606 (99h:20011)

[15] J. RickARD, Equivalences of derived categories for symmetric algebras. J. Algebra 257(2002), 460-481. MR1947972 (2004a:16023)

[16] C.C. XI, Representation dimension and quasi-hereditary algebras. Adv. in Math. 168(2002), 193-212. MR.1912131 (2003h:16015)

[17] C.C. XI, On the finitistic dimension conjecture I: related to representation-finite algebras. J. Pure Appl. Alg. 193(2004), 287-305. MR2076389

School of Mathematical Sciences, Beijing Normal University, 100875 Beijing, PeoPLE's Republic of China

E-mail address: liuym2@263.net 